\title{
Ancora sulle onde interne nel lago di Bracciano e sui fenomeni ad esse collegati $(*)$
}

\author{
P. Caloi, M. Migani, G. Pannocchia
}

Ricevuto il 31 Luglio 1961

1. - E stata provata l'esistenza ( $\left.{ }^{1}\right)$ di onde interne, legate alla termodinamica del lago di Bracciano. Restava da registrare, a riprova dell'origine di tali oscillazioni, le contemporanee variazioni di temperatura, che dovevano verificarsi in corrispondenza della zona del salto termico.

A tale scopo sul lago di Bracciano furono messi in funzione un batitermografo Askania, un limnografo a galleggiante costruito a cura dell'I.N.G. e un termoigrografo S.I.A.P.

Queste apparecchiature vennero istallate in località "La casina Bianca " nel comune di Trevignano sul lago.

Il batitermografo ha funzionato dal 9 Ottobre 1959 fino al 9 Novembre 1960, il termoigrografo dal 4 Maggio 1960 al 31 Ottobre 1960, il limnografo dall'11 Maggio 1960 al 5 Novembre 1960.

Il funzionamento degli strumenti, per varie ragioni, non fu sempre continuo.

Il limnografo, fissato ad un palo infitto nel fondo del lago, venne messo ad una distanza dalla riva di una ventina di metri; esso era del consueto tipo a galleggiante, in tubo con ammissione d'acqua a mezzo di valvola regolabile, collegato a leva d'ingrandimento, registrazione settimanale.

Il termoigrografo fu messo con i consueti accorgimenti usati nelle misure meteorologiche; anh'esso era a registrazione settimanale.

Il batitermografo Askania è costituito da una parte sensibile $\mathrm{S}$ (figg. $2,3,4$ ), la quale viene immersa a varie profondità nell'acqua a mezzo del cavo $\mathrm{C}$, che la collega agli strumenti di misura; in essa vi è un termometro a resistenza variabile con la temperatura $T$ ed un tubo Bourdon, che agisce su una resistenza variabile, per misurare la pressione

(*) Questo lavoro è stato compiuto con contributi del Consiglio Nazionale delle Ricerche. 


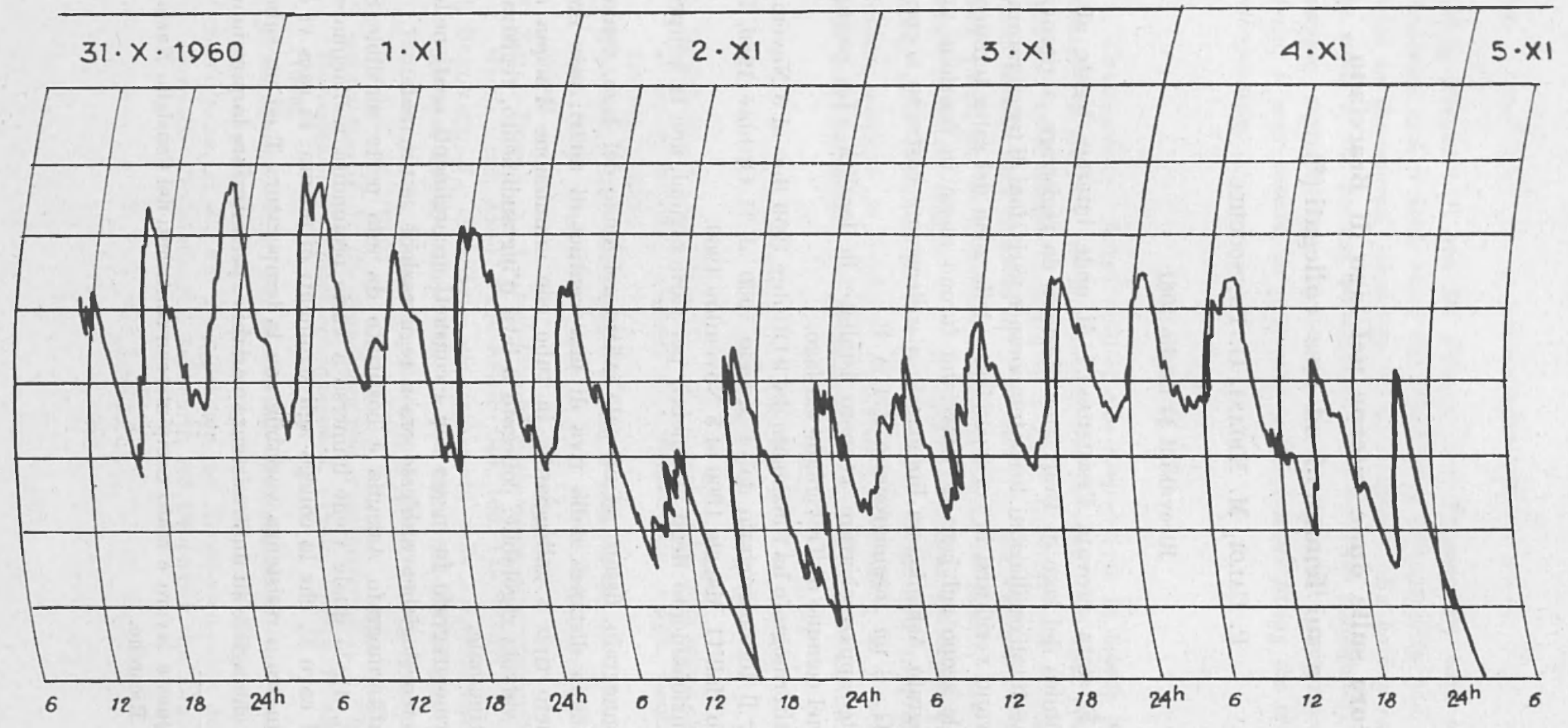

Fig. 1 - Chiari esempi di onde interne, registrate alla superficie del lago di Bracciano, in concomitanza con le ampie oscillazioni della superficie del salto termico 


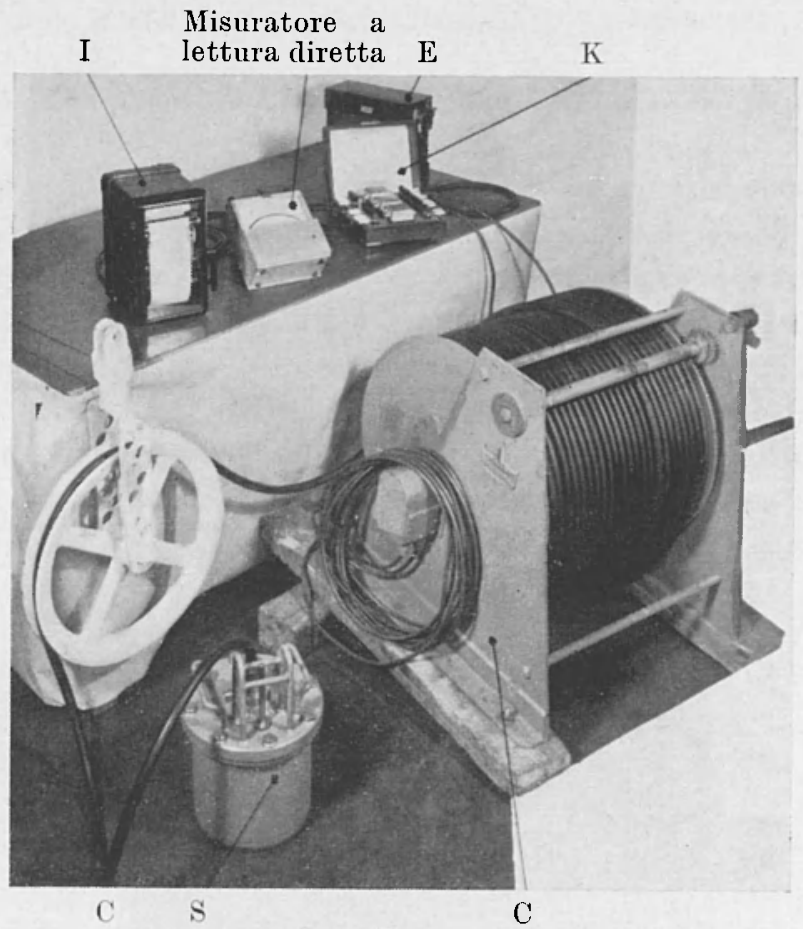

Fig. 2

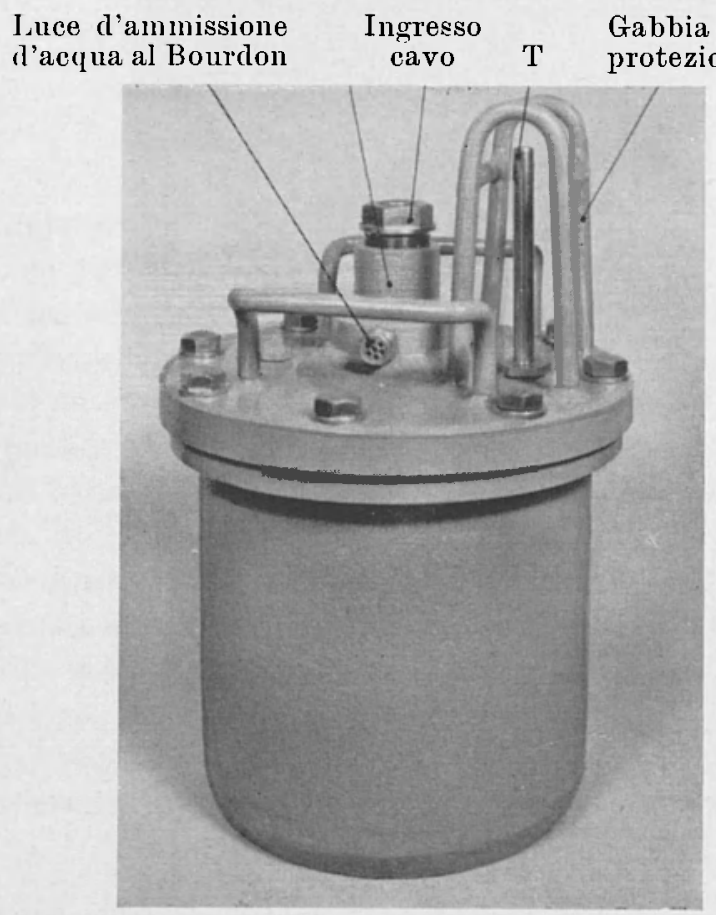

Fig. 3 - Elemento sensibile $S$, veduta d'insieme 


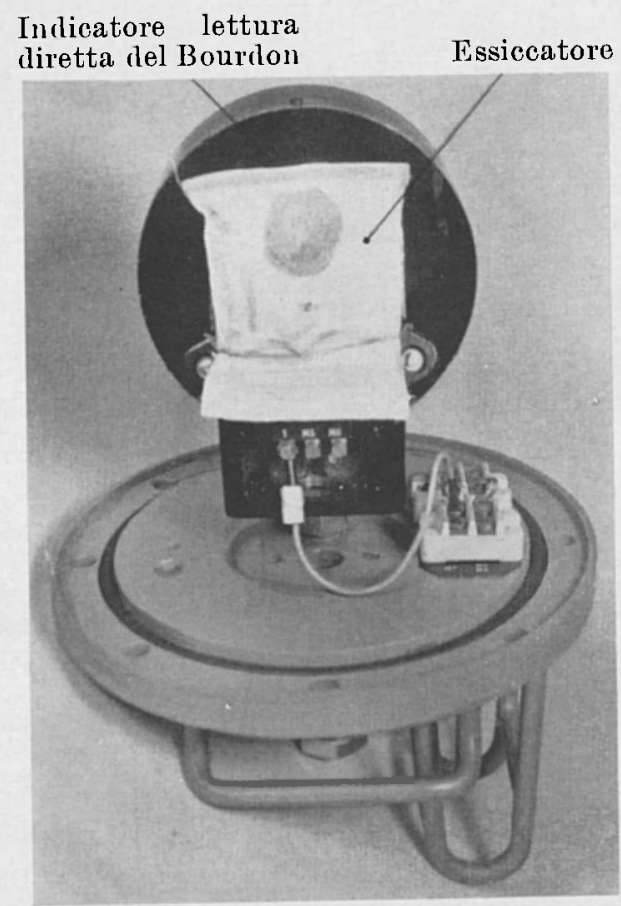

Fig. 4 - Interno dell'elemento sensibile S 
idrostatica alle varie profondità e quindi la profondità stessa in metri d'acqua.

Sia il termometro a resistenza variabile, sia la resistenza comandata dal tubo Bourdon sono in comunicazione elettrica a mezzo del cavo $\mathrm{C}$, ed attraverso la cassetta di connessioni $\mathrm{K}$, con l'apparato registratore e più precisamente con un indicatore-marcatore a bobine incrociate I (allo scopo di rendere le misure indipendenti, entro certi limiti, dalle variazioni di tensione della batteria $\mathrm{E}$ inserita nel circuito). In questo strumento, come è noto, l'equipaggio rimane nella posizione di zero prescelta fino a quando le intensità di corrente nelle due bobine sono

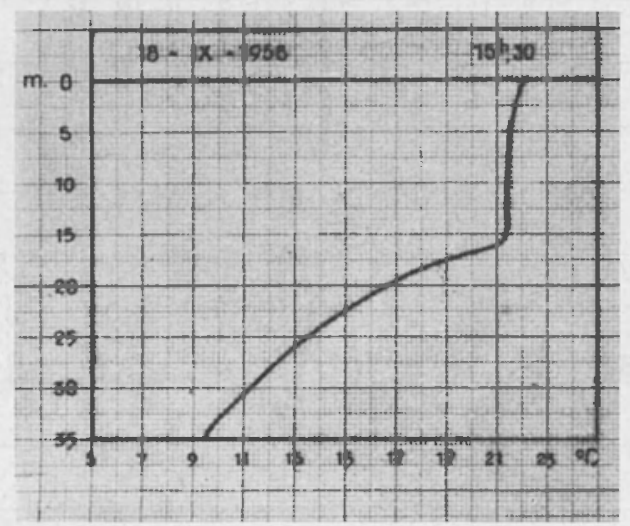

Fig. 5

le stesse. Questo indicatore-marcatore alternativamente ̀̀ collegato, a mezzo di un commutatore comandato dallo stesso movimento ad orologeria che fa camminare la carta di registrazione, al termomento ed al tubo Bourdon e batte (sempre per via meccanica comandata dal detto movimento ad orologeria) un punto blu ed uno rosso. In tal modo sulla carta, la quale avanza di $2 \mathrm{~cm}$ l'ora, graduata in gradi Celsius ed in metri d'acqua, si hanno le due curve rispondenti alle variazioni termiche e di profondità.

La testa sensibile è stata calata in acqua a circa $150 \mathrm{~m}$ dalla riva, è stata tenuta immersa, normalmente, ad una profondità di circa $16 \mathrm{~m}$ (ciò è stato realizzato facendo uso di una adeguata boa, ancorata al fondo del lago, alla quale era appeso lo strumento a mezzo di una carrucola; il cavo, lungo il fondo del lago, portava le indicazioni allo strumento di misura, sito sulla riva in un casotto in muratura). In giorni 
diversi sono state eseguite colonne di scandaglio, cioè innalzamenti o abbassamenti della testa sensibile di quantità note.

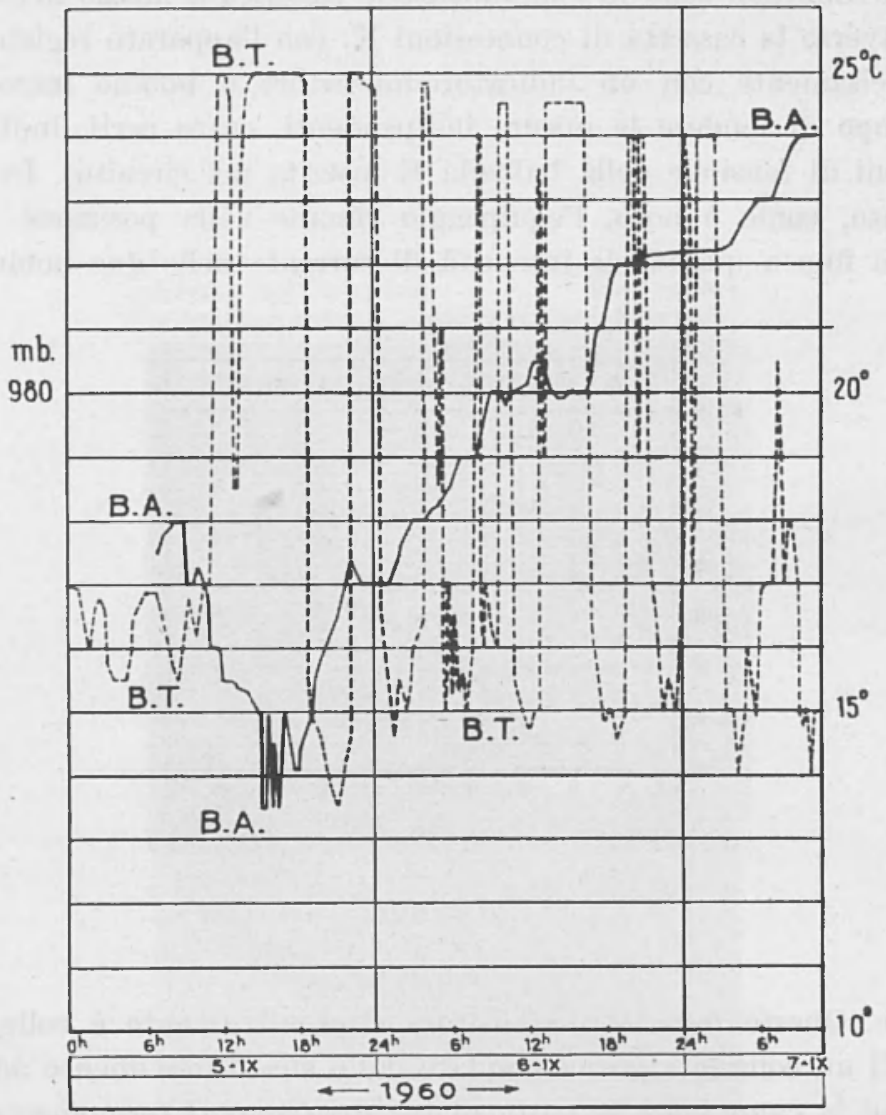

Fig. 7 - Ampie oscillazioni della temperatura, registrate alla profondità di 16 metri ca dal hatitermografo (B. T.), in relazione con il passaggio di una serie di temporali sul lago di Bracciano, associati alle variazioni di pressione diagrammate in $\mathrm{B}$. A.

Tanto i risultati delle registrazioni che delle colonne di scandaglio sono riportati qui di seguito ed illustrati; mancano i dati di registrazione delle profondità in quanto le variazioni eseguite non rientrano nell'ambito della sensibilità dello strumento; tuttavia le profondità furono determinate accuratamente per mezzo di misurazione diretta. 

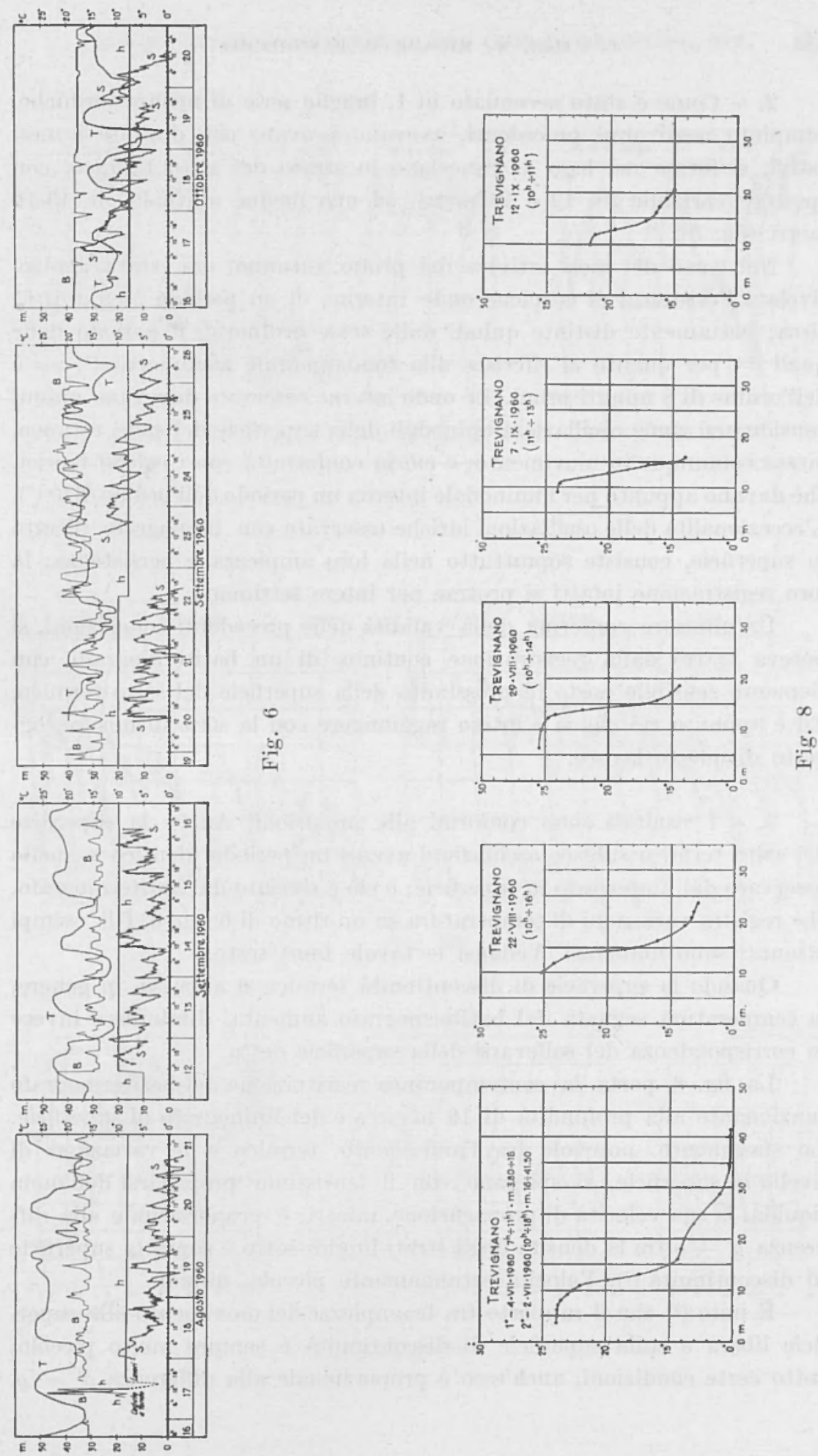
2. - Come è stato accennato in 1 , lunghe serie di misure termiche, compiute negli anni precedenti, avevano provato che durante $i$ mesi estivi, si forma nel lago di Bracciano lo strato del salto termico, con spessore variabile fra 12 e 18 metri, ed una media abituale di 15-16 metri (fig. 5).

Nel corso dei mesi estivi e del primo autunno, era stata inoltre rivelata l'esistenza di cospicue onde interne, di un periodo medio di $6^{\text {h }}$ circa; nettamente distinte quindi dalle sesse ordinarie, il periodo delle quali - per quanto si riferisce alla fondamentale asimmetria $\left(^{2}\right)$ - è dell'ordine di 8 minuti primi. Le onde interne osservate dovevano quindi considerarsi come oscillazioni uninodali della superficie del salto termico, messa comunque in movimento; e ciò in conformità con i calcoli teorici, che davano appunto per l'uninodale interna un periodo dell'ordine di $6^{\text {h }}\left(^{1}\right)$. L'eccezionalità delle oscillazioni idriche osservate con limnografo filtrato in superficie, consiste soprattutto nella loro ampiezza e persistenza: la loro registrazione infatti si protrae per intere settimane.

Un'ulteriore conferma della validità delle precedenti conclusioni, si poteva trarre dalla registrazione continua di un batitermografo, con elemento sensibile posto in prossimità della superficie del salto termico. Ed è appunto ciò che si è inteso raggiungere con la serie di misure, oggetto di questo lavoro.

3. - I risultati sono conformi alle previsioni. Anche la superficie del salto termico subisce oscillazioni aventi un periodo identico a quello osservato dal limnografo in superficie; e ciò è rivelato dal batitermografo, che registra variazioni di temperatura su un ritmo di $6^{\text {h }}$ circa. Gli esempi ottenuti sono numerosi. Vedansi le tavole fuori testo.

Quando la superficie di discontinuità termica si abbassa, in genere, la temperatura segnata dal batitermografo aumenta; diminuisce invece in corrispondenza del sollevarsi della superficie detta.

La fig. 6 porta la contemporanea registrazione del batitermografo funzionante alla profondità di $16 \mathrm{~m}$ circa e del limnografo di superficie. Lo sfasamento notevole fra l'andamento termico e le variazioni di livello in superficie, si spiegano con il lentissimo propagarsi dell'onda liquida: la sua velocità di propagazione, infatti, è proporzionale alla diferenza $\varrho^{\prime}-\varrho$ fra la densità degli strati liquidi sotto e sopra la superficie di discontinuità $\left({ }^{3}\right)$. Velocità estremamente piccola, quindi.

È noto $\left({ }^{3}\right)$ che il rapporto fra le ampiezze del movimento alla superficie libera e sulla superficie di discontinuità è sempre molto piccolo; sotto certe condizioni, anch'esso è proporzionale alla differenza $\varrho^{\prime}-\varrho$. 

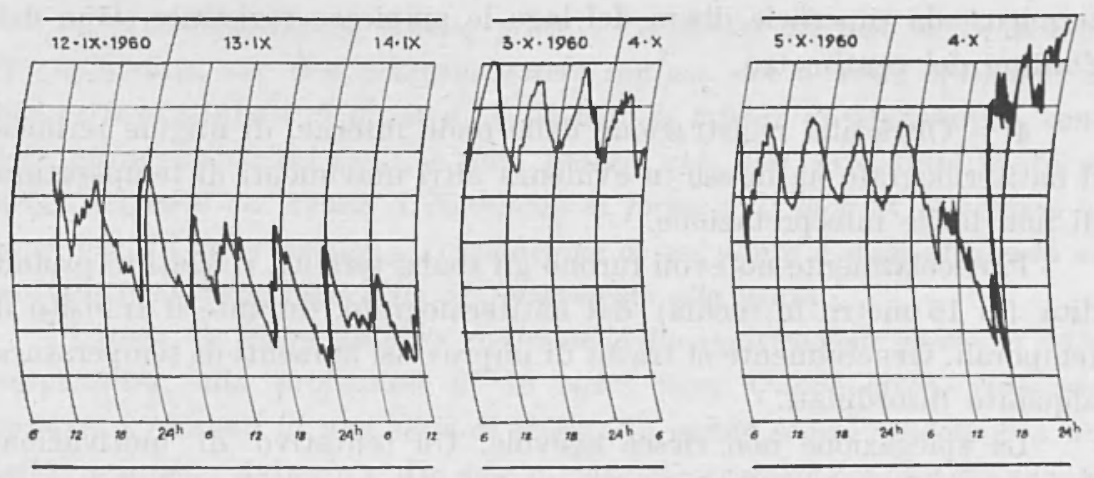

Fig. 9a
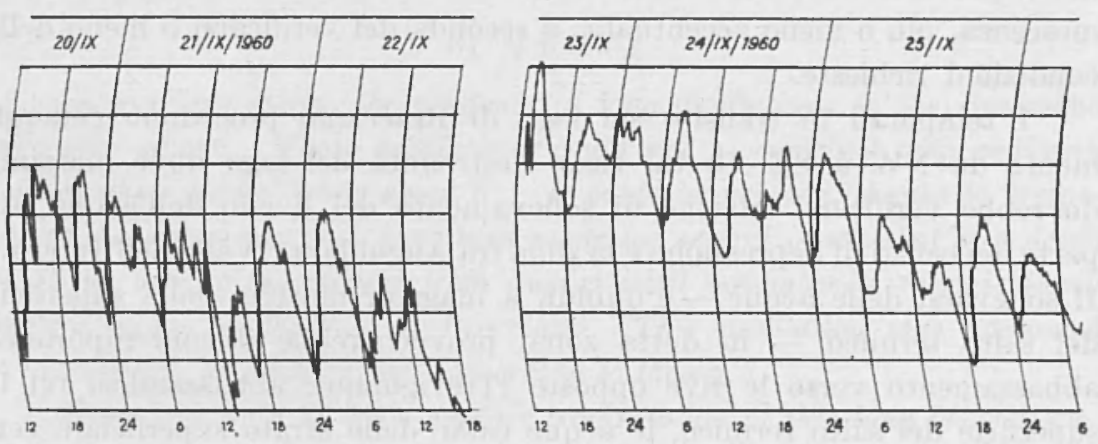

Fig. $9 b$
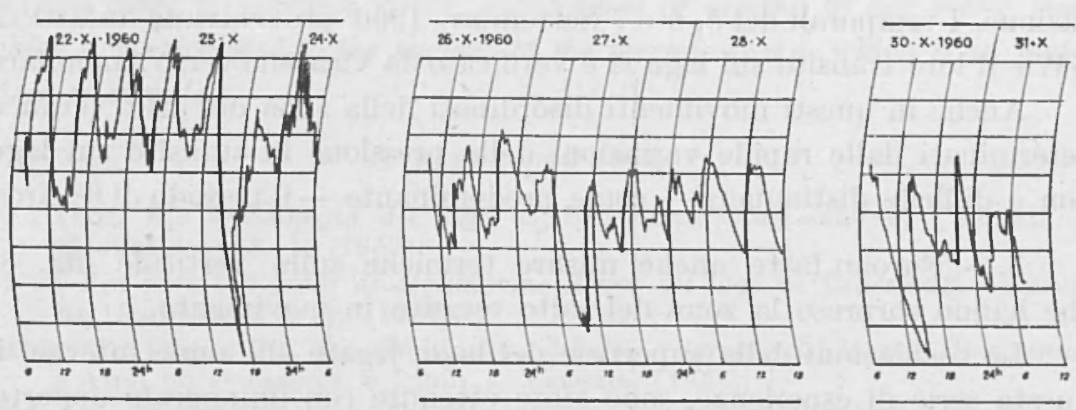

Fig. 9c 
Ciò spiega perchè, malgrado gli ampi movimenti della superficie del salto termico, alla superficie libera del lago le ampiezze registrate siano dell'ordine del centimetro.

4. - Oltre alla registrazione delle onde interne, di origine termica, il batitermografo ha messo in evidenza altri movimenti di temperatura, di non facile interpretazione.

Particolarmente notevoli furono gli sbalzi termici, rivelati in profondità (di 16 metri, in media) dal batitermografo, durante il transito di temporali. Generalmente si tratta di improvvisi aumenti di temperatura, alquanto disordinati.

La spiegazione non riesce agevole. Un tentativo di motivazione potrebbe trovarsi nell'azione meccanica dei centri di depressione in transito sul lago. E noto che, quando un disturbo microbarico si muove sopra una massa liquida, sotto certe condizioni di direzione e di velocità $\left({ }^{4}\right)$, provoca verso l'estremità chiusa del canale, golfo o lago, un'intumescenza, più o meno accentuata, a seconda del verificarsi o meno delle condizioni richieste.

I temporali in transito sul lago di Bracciano procedono generalmente da NW a SE. In tal caso, l'estremità del lago dove massima dovrebbe verificarsi l'azione di sollevamento del livello dell'acqua, da parte dei centri di depressione è la zona fra Anguillara e Valle dell'Inferno. Il sollevarsi delle acque - e quindi, a maggior motivo, della superficie del salto termico - in detta zona, provocherebbe il contemporaneo abbassamento verso le rive opposte (Trevignano): abbassandosi ivi la superficie del salto termico, le acque calde dello strato superficiale verrebbero convogliate verso il batitermografo, provocando l'aumento termico osservato.

Gli esempi riportati (fig. 7) sembrano confermare la tentata spiegazione. I temporali del 5, 6 e 7 Settembre 1960 provenivano infatti da NW e il loro transito sul lago si è verificato da Vicarello verso Anguillara.

Anche in questi movimenti disordinati della zona del salto termico, determinati dalle rapide variazioni della pressione in transito sul lago, non è difficile distinguere - come predominante - il periodo di $6^{\text {h }}$ circa.

5. - Furono fatte anche misure termiche sulla verticale (fig. 8), che hanno sorpreso la zona del salto termico in movimento.

Le oscillazioni della superficie del lago, legate alle onde interne, in questa serie di esperienze, sono state ottenute con limnografo opportunamente filtrato. Gli esempi ottenuti sono risultati - come in precedenti indagini - d'estrema chiarezza (figg. 1, 9a, 9b, 9c). 


\section{RIASSUNTO}

In precedenti lavori, è stata provata l'esistenza nel lago di Bracciano di oscillazioni, che non potevano essere confuse con le sesse ordinarie: il loro periodo è infatti di $6^{h}$ circa. Sulla base di misure di temperatura, condotte attraverso alcuni anni, ̀̀ stato provato che, alla profondità $d i 15 \mathrm{~m}$ circa, nei mesi da Agosto a Novembre si forma, nel lago di Bracciano la superficie del salto termico. L'oscillazione di cui sopra è stata attribuita ai movimenti di detta superficie, conformemente alla teoria.

Restava da trarre un'altra conferma dalla registrazione continua della temperatura, alla profondità di 16 metri circa. Questo lavoro riassume appunto $i$ risultati di una serie di misure in questo senso, condotte con un batitermografo "Askania". Da esse appare chiaramente confermato quanto si era ottenuto nei precedenti lavori: le oscillazioni di $6^{\text {h }}$ circa sono appunto quelle associate alla superficie del salto termico, comunque messa in movimento.

\section{$S U M M A R Y$}

In previous works, the existence of long oscillations in the Bracciano lake was proved. These oscillations could not be confused with ordinary seiches, their period being about $6^{h}$. It could be prooved, thanks to temperature measurements wich have been made for several years, that at a depth of $15 \mathrm{~m}$, during the months from august until november, a thermic jump surface occurs in the lake of Bracciano. This oscillation was attributed to movements on such surface, according to theory.

There was only to draw another confirmation of this long lasting temperature registration at a depth of around $16 \mathrm{~m}$. In fact, the present work gives a summary of the results of a series of measures in this sense, made with a batithermograph "Askania". The results thus obtained clearly confirm those of previous works: oscillations of around $6^{\text {h }}$ are really those which are associated to the surface of the thermic jump, which is in movement at any rate.

\section{BIBLIOGRAFIA}

(1) Calor, P., Termologia del lago di Bracciano: onde interne, "Annali di Geofisica ", 12, 1, (1959).

(2) - Sui possibili modi di oscillazione libera del lago di Bracciano, "Annali di Geofisica ", 3, 1, (1950).

Caloi, P., Peronaci, F., Oscillazioni libere e forzate del lago di Bracciano, "Arch. di Oceanogr. e Limn. ", Venezia, (1958).

( $\left.{ }^{3}\right)$ LAMB, H., Hydrodynamics, VI ediz., pag. 370 e ss., (1932).

${ }_{\left({ }^{4}\right)}$ CaloI, P., Sesse dell'alto Adriatico, con particolare riguardo al golfo di Trieste. "R. Comit. Talass. It., Mem.", CCXLVII, (1938). 
Nelle tavole che seguono sono riportati, opportunamente ridotti.

essi appare chiaro che le oscillazioni predominanti sono quelle che

susseguono con un ritmo di $6^{\text {h }}$ cirean, coperiodali con le analoche oseit lazioni idriche di superficie (figg. 1, 9). Nella III tavola sono pure ri-

portati i bruschi sbalzi di temperatura, che si osservano in profondità,

durante il passaggio di temporali sul lago
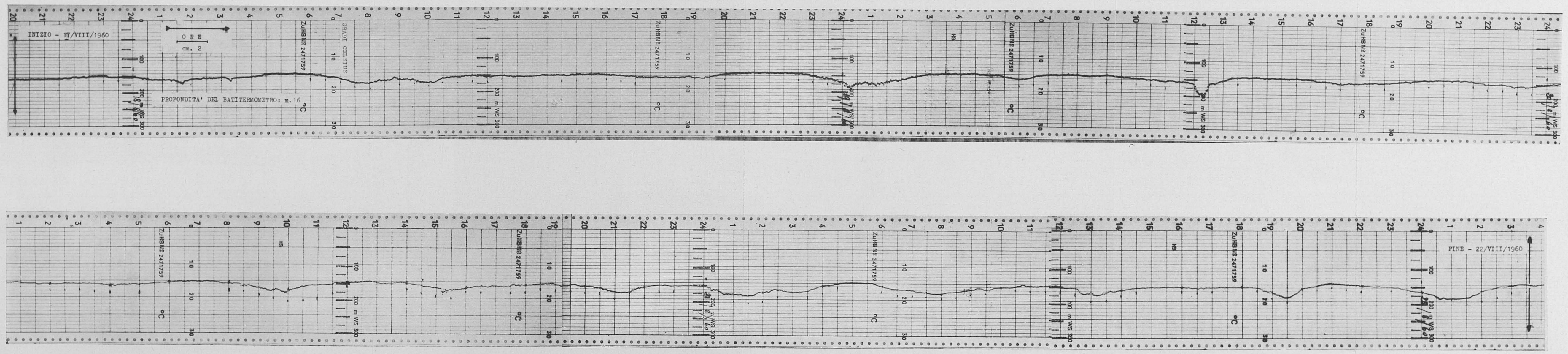

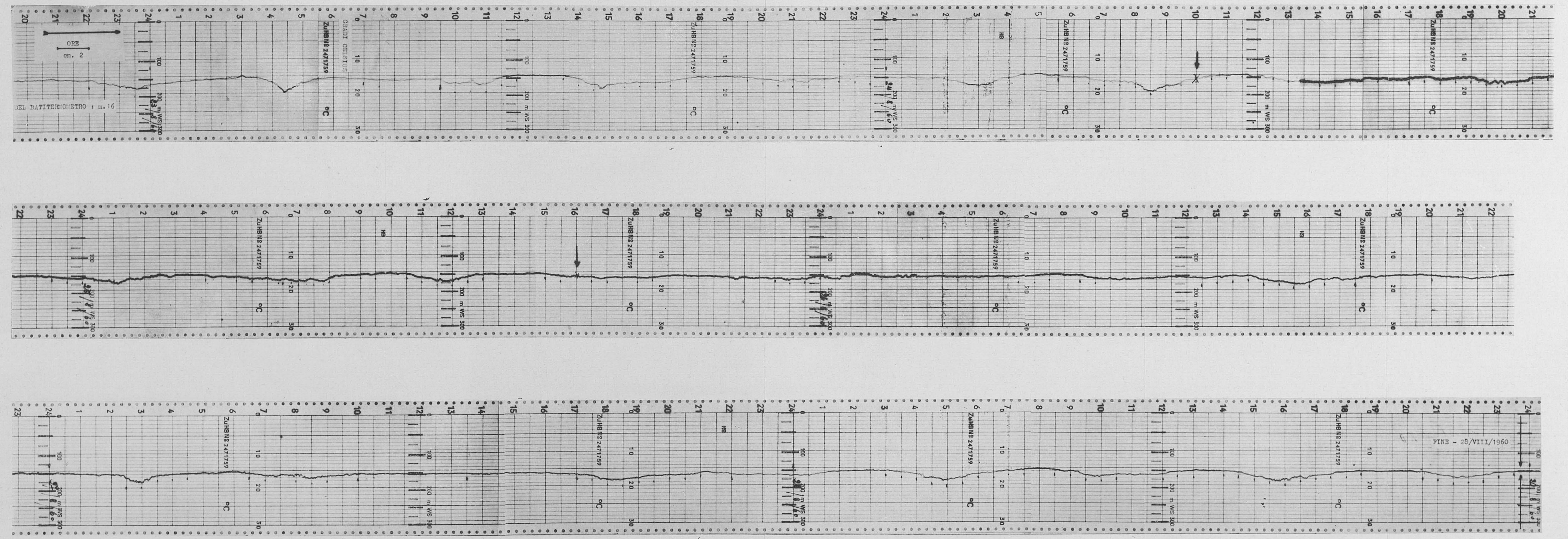

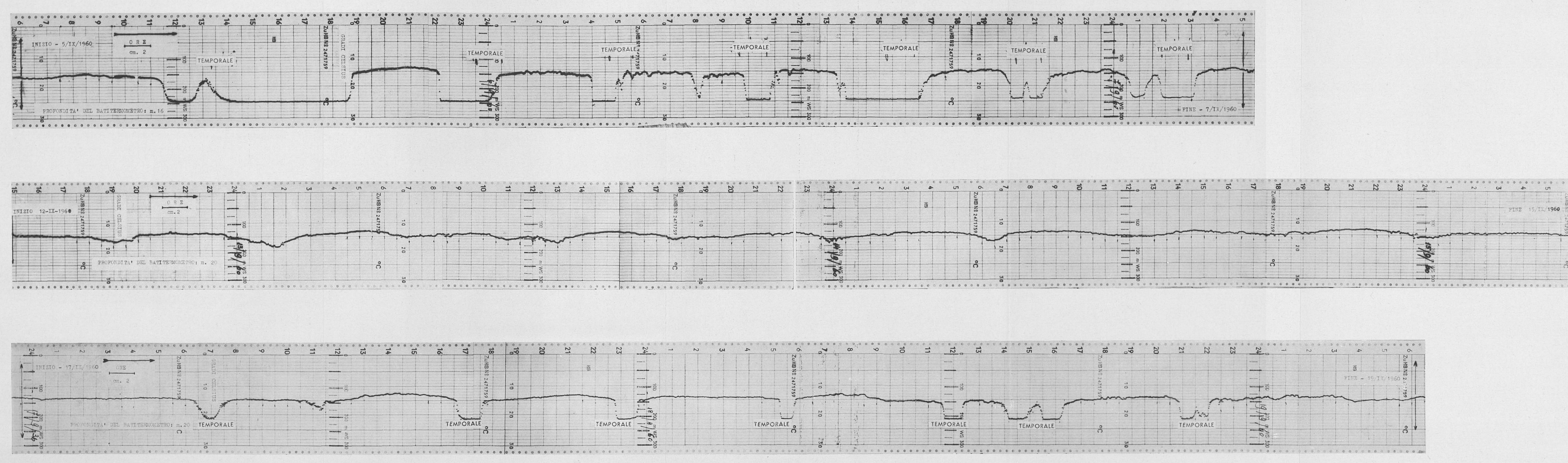

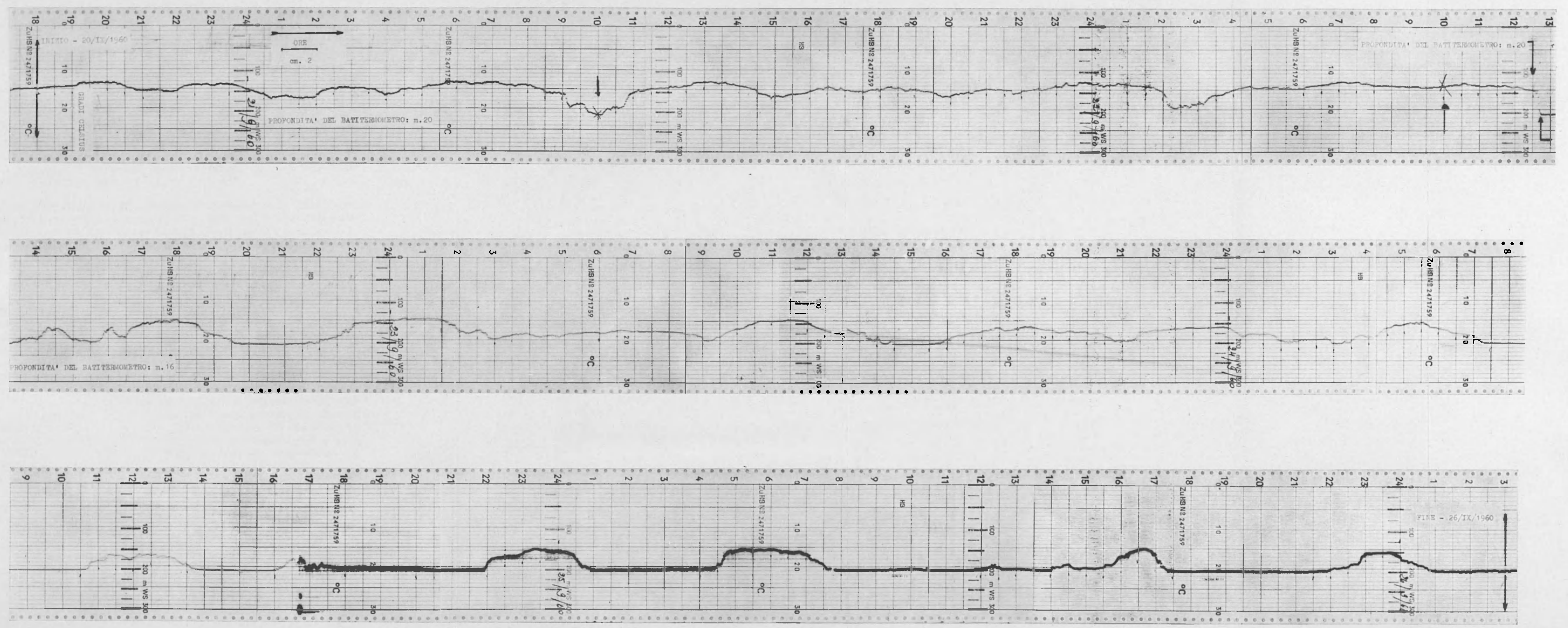